\title{
NHS England-funded CT fractional flow reserve in the era of the ISCHEMIA trial
}

\author{
Authors: Hazhee Rasoul, ${ }^{\text {A }}$ Saad Fyyaz, ${ }^{\mathrm{B}}$ Daniela Noakes, ${ }^{\mathrm{C}}$ Carl Shakespeare, ${ }^{\mathrm{D}}$ Sarojini David, ${ }^{\mathrm{E}}$ Zeeshan M Khawaja, ${ }^{\mathrm{D}}$ \\ Nikolaos Papamichail ${ }^{\mathrm{D}}$ and Khaled Alfakih ${ }^{\mathrm{F}}$
}

\section{Background}

The National Institute for Health and Care Excellence (NICE) 2016 guidelines (CG95) recommend patients with new stable chest pain be investigated with computed tomography coronary angiography (CTCA). An updated guideline (MTG32) recommended using $\mathrm{CT}$ fractional flow reserve (CTFFR) as a gatekeeper to invasive coronary angiography (ICA) for patients with coronary stenosis on CTCA. Subsequently, NHS England negotiated a UK-wide contract with HeartFlow, the provider of CTFFR. We describe our experience with CTFFR and consider the impact of the recent ISCHEMIA trial on these guidelines.

\section{Methods}

We prospectively collected ICA and revascularisation data on all patients undergoing CTFFR from January 2019 to March 2020.

Results

One-hundred and twenty-five of 140 patients completed CTFFR analysis. Eighty-one patients had CTCA stenosis $>50 \%$. Thirty-six had positive CTFFR; 29 underwent ICA with 22 (75.9\%) revascularised. Forty-five had negative CTFFR; 14 underwent ICA and four (28.6\%) were revascularised. The average cost of investigation per patient (PP) was $£ 971.95$. Had these patients undergone ICA directly with no functional test after CTCA, the average cost would be $£ 932.51$ PP.

\section{Conclusion}

Our revascularisation rates suggest that CTFFR can potentially be a gatekeeper to ICA but does not necessarily yield cost savings.

KEYWORDS: guidelines, stable chest pain, CT coronary angiography, $\mathrm{CT}$ fractional flow reserve, audit

DOI: 10.7861/clinmed.2020-0691

Authors: Afoundation year-2 doctor, Lewisham and Greenwich NHS Trust, London, UK; ${ }^{\mathrm{B}}$ specialty registrar in cardiology, Lewisham and Greenwich NHS Trust, London, UK and St George's University Hospitals NHS Trust, London, UK; ${ }^{C}$ specialty registrar in cardiology, Lewisham and Greenwich NHS Trust, London, UK; ${ }^{\text {D }}$ consultant cardiologist, Lewisham and Greenwich NHS Trust, London, UK; ${ }^{\text {E}}$ consultant radiologist, Lewisham and Greenwich NHS Trust, London, UK;

F consultant cardiologist, Lewisham and Greenwich NHS Trust, London, UK and honorary senior lecturer, King's College London, London, UK

\section{Background}

New stable chest pain is a common presentation and can be investigated with a variety of diagnostic modalities including ischaemia tests such as stress echocardiography (SE), or anatomical assessment with invasive coronary angiography (ICA), or more recently, computed tomography coronary angiography (CTCA). ${ }^{1}$ The UK National Institute for Health and Care Excellence (NICE) guidelines in 2016 recommended that all patients with new onset stable chest pain should be investigated with CTCA as a first-line test. ${ }^{2}$ CTCA has an excellent negative predictive value of $99 \%$ and high sensitivity of $95 \%$ for detection of coronary artery disease (CAD) when compared with ICA and is a relatively low-cost noninvasive test with a UK NHS tariff of $£ 220$.

When a patient is found to have a severe coronary stenosis on CTCA, routine clinical practice is for the patient to undergo ICA to confirm the presence of a severe stenosis and often undertake invasive fractional flow reserve (FFR), which measures pressure difference before and after a coronary artery stenosis, using a pressure wire inserted into the coronary artery and pharmacological vasodilator stress to calculate a ratio. An FFR of $<0.80$ is considered significant and the patient would usually undergo revascularisation, normally within the same procedure. ICA and invasive FFR are relatively expensive (the NHS tariff for ICA is $£ 1,000$ and the invasive FFR is an additional $£ 336$ ) and carry a small risk, hence should be used judiciously.

CTCA can be associated with false positive results, whereby it overestimates the degree of coronary stenoses compared with ICA. ${ }^{3}$ Importantly, studies assessing the sensitivity and specificity of CTCA against ICA were undertaken in patient populations with low-intermediate prevalence of $C A D .{ }^{4}$ A recent meta-analysis of outcomes of CTCA and stress tests confirmed that the use of CTCA in patients with low-risk acute chest pain and stable chest pain is associated with a higher rate of use of ICA, and a higher rate of revascularisation, with no evidence that outcomes are improved. ${ }^{5}$ Hence, expanding the use of CTCA as first-line test to all patients, including those with higher prevalence of $C A D$, may lead to more downstream ICA and consequently higher healthcare costs. NICE issued a medical technology guideline in 2017 (MTG32) recommending the use of CT fractional flow reserve (CTFFR), which is a novel method applying computational fluid dynamics to derive the FFR from the CTCA data. ${ }^{6}$ CTFFR was priced at $£ 700$ per scan, but its use was endorsed following NICE assessment of health economics data from the PLATFORM trial demonstrating it to be cost effective, with fewer patients undergoing ICA following 
CTFFR. ${ }^{7}$ In 2018, NHS England negotiated a UK-wide central contract with the provider of CTFFR (HeartFlow) to cover the cost of CTFFR for cardiac CT departments which undertake high volumes of CTCA. The price of CTFFR for the NHS was recently reduced to $£ 530$. We describe our initial experience with CTFFR to assess its clinical efficacy and we undertake cost analyses to simulate different clinical pathways.

\section{Methods}

Between January 2019 and March 2020, 140 patients underwent CTFFR at Lewisham and Greenwich NHS Trust. Fifteen (10.7\%) CTCAs were rejected due to inadequate image quality. One-hundred and twenty-five patients were analysed ( 80 men; mean age 59 years).

All CTCAs were performed with a 64 slice LightSpeed VCT XTe GE scanner (GE Healthcare), using a commercially available protocol (SnapShot Pulse, GE healthcare). The scanning parameters were slice acquisition $64 \times 0.625 \mathrm{~mm}$, SFOV Cardiac, Z-axis detector coverage $40 \mathrm{~mm}$ and gantry rotation time of $350 \mathrm{~ms}$. For adapted tube voltage, small patients required $100 \mathrm{kV}$ while $120 \mathrm{kV}$ was used for all other patients. Prospective gating was used as the standard acquisition protocol. A prospectively gated calcium score scan (gantry rotation time of $350 \mathrm{~ms}, 120 \mathrm{kv}$ and $150 \mathrm{~mA}$ ) is undertaken as part of the CTCA protocol. Patients were beta-blocked aiming to achieve a heart rate of $<60$ beats per minute. CTCA were reported by a cardiologist or a radiologist. The severity of coronary stenoses was classified, based on visual assessment as severe if $>70 \%$ (or $>50 \%$ in the left main stem), moderate if $>50-70 \%$ stenosis and mild if $30-50 \%$ stenosis.

All patients with moderate or severe coronary stenoses, and some with mild stenoses, were referred for CTFFR with the CTCA data being sent to HeartFlow electronically. Coronary vessels with CTFFR $<0.8$ were deemed clinically significant, unless if this was in the distal vessels, in which case, they were not considered significant as per Heartflow recommendations. All results were reviewed by a consultant cardiologist. We prospectively collated data on these patients including CTFFR result, subsequent investigations, and revascularisation.

Patients were separated into those with $<50 \%$ stenosis and those with $>50 \%$ stenosis on CTCA. The reason for this is that the European Society of Cardiology (ESC) guidelines 2019 recommend patients with $>50 \%$ stenosis on CTCA should have functional testing with CTFFR or imaging stress tests. HeartFlow recommends that patients with $30-50 \%$ stenosis on CTCA should also have CTFFR as there are a very small proportion of those patients where the stenosis can be significant. ${ }^{8}$

A cost analysis was undertaken using the following NHS tariffs: CTCA $£ 220$, CTFFR $£ 530$, ICA $£ 1,000$, invasive FFR $£ 336$ ( $£ 279$ pressure wire $+£ 57$ catheter laboratory time). ${ }^{9}$

\section{Results}

One-hundred and twenty-five of the 140 patients had their CTFFR completed. Thirty-seven (30\%) patients had a positive CTFFR $(<0.8)$ in one of more vessels and 29 were referred for ICA. Eightyeight $(70 \%)$ patients had a negative CTFFR and 15 underwent ICA overall (Fig 1).

\section{CTFFR in patients with mild CTCA stenosis (30-50\%)}

Forty-four patients had mild stenosis ( $30-50 \%$ stenosis) on CTCA and went on to have CTFFR. Forty-three had negative CTFFR, three of which also underwent subsequent SE (all negative). One patient had a positive CTFFR but did not attend for further investigation with SE (Table 1).

\section{CTFFR in patients with $>50 \%$ CTCA stenoses}

Eighty-one patients had moderate or severe stenosis ( $>50 \%$ stenosis) on CTCA and underwent CTFFR.
Fig 1. Downstream investigations and outcomes of patients following computed tomography fractional flow reserve analysis. $\mathrm{CT}=$ computed tomography; $\mathrm{CTCA}=\mathrm{CT}$ coronary angiography; $C T F F R=C T$ fractional flow reserve; DNA = did not attend; ICA = invasive coronary angiography; $\mathrm{SE}=$ stress echocardiography.

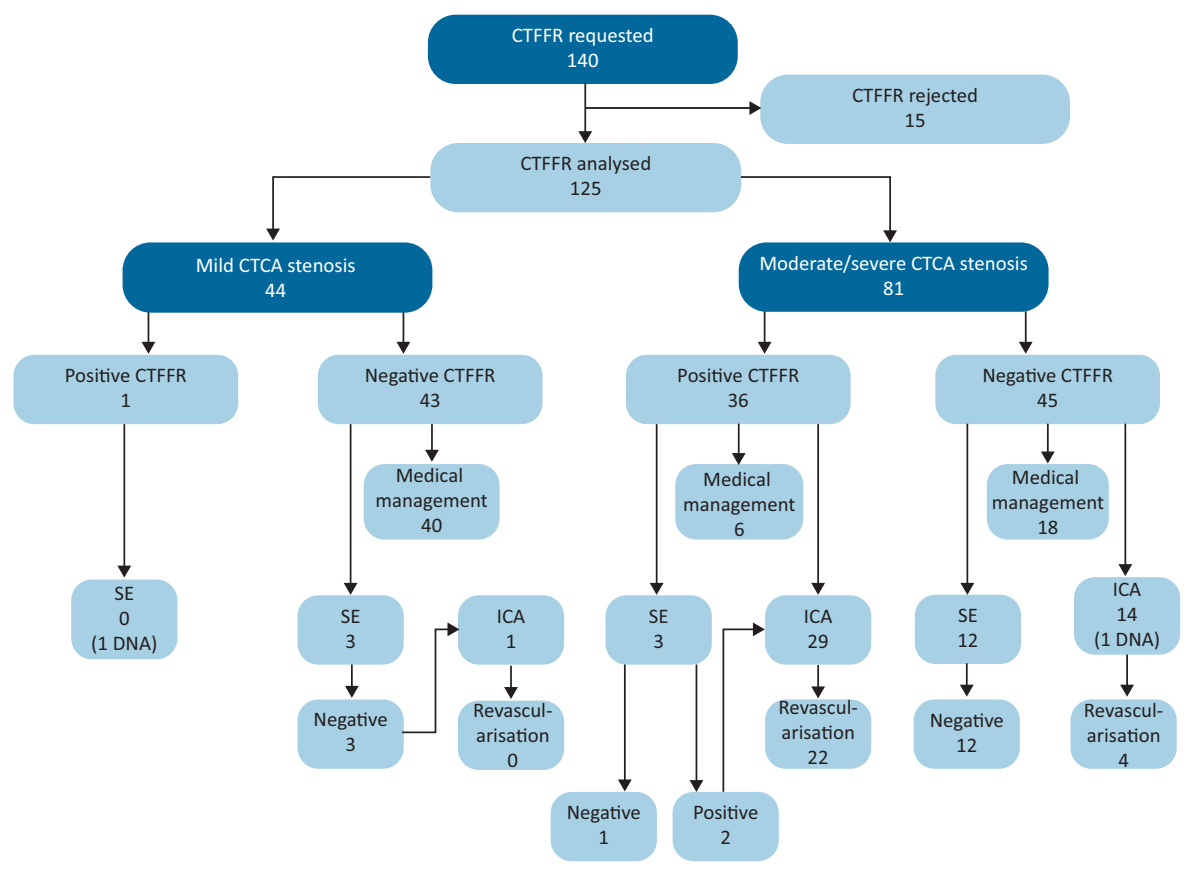


Table 1. Number of patients undergoing medical management, stress echocardiography, invasive coronary angiography or revascularisation in our cohort of patients

\begin{tabular}{|c|c|c|c|c|c|c|c|c|}
\hline & \multirow[b]{2}{*}{ Total } & \multicolumn{3}{|c|}{ Investigation } & \multicolumn{4}{|c|}{ Revascularisation } \\
\hline & & $\begin{array}{l}\text { Medical } \\
\text { management }\end{array}$ & SE negative & SE positive & ICA & PCI & CABG & DNA \\
\hline \multicolumn{9}{|c|}{$\begin{array}{l}\text { CTCA stenosis severity with } \\
\text { positive CTFFR }\end{array}$} \\
\hline Mild & 1 & 0 & 0 & 0 & 0 & 0 & 0 & 1 \\
\hline \multicolumn{9}{|c|}{$\begin{array}{l}\text { CTCA stenosis severity with } \\
\text { negative CTFFR }\end{array}$} \\
\hline Mild & 43 & 40 & 3 & 0 & 1 & 0 & 0 & 0 \\
\hline Moderate/severe & 45 & 18 & 12 & 0 & 14 & 4 & 0 & 1 \\
\hline
\end{tabular}

\section{Positive CTFFR}

Thirty-six patients had a positive CTFFR. Six were medically managed, three had SE (two positives and one negative), 29 patients in total underwent ICA. Twenty-two (75.9\%) were found to have a severe stenosis and were revascularised. Four patients had a false positive CTFFR and were medically managed following ICA. Of the revascularised patients, five had a false negative CTFFR in one vessel but true positive CTFFR in another vessel (Fig 2). One additional patient had a false positive CTFFR in one vessel and false negative CTFFR in a different vessel. These six patients were correctly identified on a per patient basis and appropriately referred for ICA and revascularised.

\section{Negative CTFFR}

Forty-five patients had a negative CTFFR. Eighteen were medically managed, 12 were referred for SE for further reassurance (all negative), 14 underwent ICA with nine found to have non-obstructive CAD and five found to have severe stenoses (Table 2). Four of these patients were revascularised and classified as false negative CTFFR (three revascularised on angiographic appearance and one following positive invasive FFR). One of the revascularised patients had CTCA
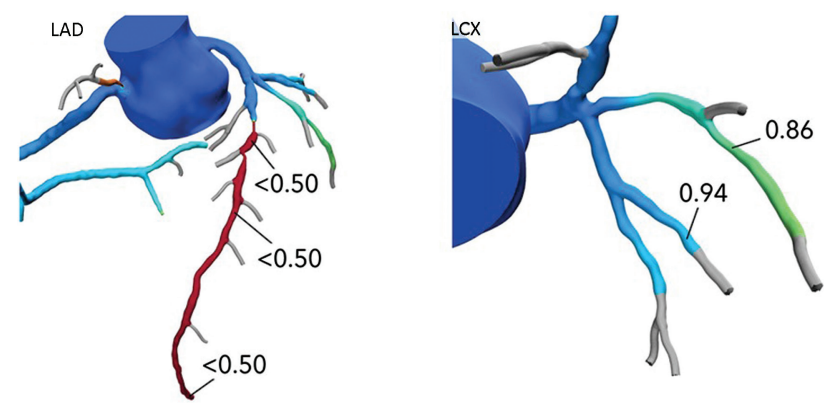

Fig 2. CTFFR model for a patient with false negative CTFFR in the intermediate vessel but true positive CTFFR in the LAD. This patient was found to have severe disease in both LAD and intermediate vessels on ICA. $\mathrm{CT}=$ computed tomography; $C T F F R=C T$ fractional flow reserve; $L A D=$ left anterior descending; LCX = left circumflex. and ICA evidence of severe stenosis, but motion artefact of that vessel precluded the CTFFR analysis in that vessel.

Therefore, four patients risked being missed based on CTFFR result alone. However, three out of the four patients had significant calcification of their vessels with calcium scores ranging $622 \mathrm{HU}$ to $1,108 \mathrm{HU}$. A high degree of coronary calcification is known to reduce the accuracy of CTFFR.

\section{CTFFR in patients with moderate $(50-70 \%)$ versus} severe stenosis $(>70 \%)$

We further subdivided the patients with $>50 \%$ stenosis into those with moderate stenosis (50-70\% stenosis on CTCA) and those with severe stenosis ( $>70 \%$ stenosis on CTCA).

\section{Moderate stenosis}

Forty-four patients had moderate stenosis (50-70\%), of which, nine had positive CTFFR and 35 negative CTFFR. In those patients with positive CTFFR, four out of the seven (57\%) who underwent ICA were revascularised. In those with a negative CTFFR, three out of the nine $(33.3 \%)$ who underwent ICA were revascularised.

\section{Severe stenosis}

Thirty-seven had severe stenosis ( $>70 \%$ stenosis), of which, 27 had positive CTFFR and 10 negative CTFFR. In those with positive CTFFR, 18 out of the 22 (82\%) patients who underwent ICA were revascularised. In those with a negative CTFFR, only one of the five patients (20\%) who underwent ICA was revascularised.

\section{Invasive FFR versus CTFFR}

Twenty-four patients underwent invasive FFR analysis of 28 vessels in total. When we compared, on a per-vessel basis, the CTFFR with the invasive FFR values, there were seven true positive and nine true negative values, so an overall concordance of $57 \%$. Seven vessels had a false positive CTFFR and five vessels had a false negative CTFFR. For the false negative vessels, CTFFR values ranged from 0.81 to 0.87 versus invasive FFR values of 0.71 to 0.79 . 
Table 2. Findings for all computed tomography fractional flow reserve patients who underwent invasive coronary angiography

\begin{tabular}{|c|c|c|c|c|c|}
\hline & Total & Normal/mild & Moderate & Severe & Revascularisation \\
\hline \multicolumn{6}{|c|}{ CTCA stenosis severity with positive CTFFR } \\
\hline Mild & 0 & 0 & 0 & 0 & 0 \\
\hline Moderate/severe & 29 & 1 & 5 & 23 & 22 \\
\hline \multicolumn{6}{|c|}{ CTCA stenosis severity with negative CTFFR } \\
\hline Mild & 1 & 1 & 0 & 0 & 0 \\
\hline Moderate/severe & 14 & 7 & 2 & 5 & 4 \\
\hline
\end{tabular}

$\mathrm{CT}=$ computed tomography; $\mathrm{CTCA}=\mathrm{CT}$ coronary angiography; $\mathrm{CTFFR}=\mathrm{CT}$ fractional flow reserve; $\mathrm{ICA}=$ invasive coronary angiography.

\section{Cost analysis}

We calculated an average cost per patient (PP) in our cohort of 125 patients of $£ 1,166.51$ based on the cost of CTCA with CTFFR, and ICA $+/-$ invasive FFR in patients who proceeded to this. Had patients with a CTCA stenosis of $<50 \%$ not been investigated, in line with ESC guidance, the average cost would be £971.95 PP (Table 3).

We simulated costs if all patients with CTCA stenosis $>50 \%$ were sent directly to ICA without any non-invasive functional assessment, with 24 patients undergoing invasive FFR as per our cohort. This would result in a cost of £932.51 PP.

We also simulated analysis if all patients with CTCA stenosis $>50 \%$ underwent SE instead of CTFFR and only those with positive SE referred for ICA and invasive FFR utilised at similar numbers to our cohort, the average cost would be £743.21 PP. Therefore, assuming SE replaced CTFFR following CTCA and reproduced identical results, the average cost saved would be $£ 228.74$ PP.

\section{Discussion}

Our results demonstrate that CTFFR can potentially be a useful tool in the assessment of the functional significance of coronary stenosis found on CTCA. As a proportion of patients undergoing ICA, revascularisation rate was $75.9 \%$ (22/29) for patients with a positive CTFFR versus $28.6 \%$ (4/14) for those with a negative CTFFR. CTFFR was recommended by HeartFlow to be used in all patients with a stenosis $>30 \%$. Our data and the ESC guidelines would suggest that patients with coronary stenoses of $30-50 \%$ on CTCA do not need any further investigation and simply need secondary prevention. Had CTFFR been restricted to patients with moderate and severe CTCA stenosis only ( $>50 \%$ stenoses), the revascularisation rate is $61.1 \%$ for patients with a positive CTFFR versus $8.9 \%$ in patients with a negative CTFFR. Importantly three of the four patients with false negative CTFFR had high calcium scores which suggests that we should either have a lower threshold for ICA in those patients as extensive calcific plaque makes CTFFR less accurate or they should be referred for imaging stress tests instead. ${ }^{10}$

The further analysis of the data into those with moderate and severe stenosis allows us to compare our current results with a separate audit of 652 patients investigated with CTCA in the same trust prior to the introduction of CTFFR. ${ }^{11}$ In the previous audit, 58 patients were found to have moderate stenosis on CTCA and SE was used as the gate keeper to ICA in some of these patients. Thirty-one per cent of patients with moderate stenosis underwent SE with only one positive SE who went on to have ICA. Sixty-two per cent of patients were referred directly to ICA. In total, $64 \%$ went on to have ICA. In comparison with our current data, 44 patients had moderate stenosis, of which, 35 had a negative CTFFR and nine had a positive CTFFR. Nine out of the negative CTFFR and seven out of the positive CTFFR patients were referred for ICA. Hence in total, $16(36.4 \%)$ went on to have ICA. Similarly, there were 34 patients with severe CTCA stenosis in the previous audit, and $97 \%$ were referred for ICA directly. In our current data, there were 37 patients with severe stenosis on CTCA with 27 positive CTFFR and 10 negative CTFFR. Twenty-two with positive CTFFR and five with negative CTFFR went on to have ICA, so overall 27 (73\%) had ICA. Therefore, CTFFR provided a better gatekeeping role than our previous practice, but it can also

\begin{tabular}{|c|c|c|c|c|c|c|c|}
\hline Scenario & CTCA, $n(£)$ & CTFFR, n ( $(£)$ & SE, $n(£)$ & ICA, $n(£)$ & $\begin{array}{l}\text { Invasive } \\
\text { FFR, } n(£)\end{array}$ & $\begin{array}{l}\text { Total } \\
\text { cost, } £\end{array}$ & $\begin{array}{l}\text { Per patient } \\
\text { cost, } £\end{array}$ \\
\hline Cohort & $125(27,500)$ & $125(66,250)$ & 0 & $44(44,000)$ & $24(8,064)$ & 145,814 & 1166.51 \\
\hline $\begin{array}{l}\text { Cohort if }<50 \% \text { stenosis } \\
\text { excluded }\end{array}$ & $125(27,500)$ & $81(42,930)$ & 0 & $43(43,000)$ & $24(8,064)$ & 121,494 & 971.95 \\
\hline CTCA + ICA directly & $125(27,500)$ & 0 & 0 & $81(81,000)$ & $24(8,064)$ & 116,564 & 932.51 \\
\hline $\mathrm{CTCA}+\mathrm{SE}+\mathrm{ICA}$ & $125(27,500)$ & 0 & $81(14,337)$ & $43(43,000)$ & $24(8,064)$ & 92,901 & 743.21 \\
\hline
\end{tabular}

$\mathrm{CT}$ = computed tomography; $\mathrm{CTCA}=\mathrm{CT}$ coronary angiography; $\mathrm{CTFFR}=\mathrm{CT}$ fractional flow reserve; $\mathrm{ICA}=$ invasive coronary angiography; $\mathrm{SE}=$ stress echocardiography. 
be argued that if we utilised SE more, we could have achieved similar result. It is also important to consider the advantage of CTFFR permitting assessment with a single patient visit, which is particularly pertinent in the COVID-19 era and negates the inherent delays between multiple tests. One of the caveats to this, however, is a CTFFR rejection rate of just over $10 \%$ in our data, which was after local selection where only very good quality CTCA were sent for CTFFR analysis. We note the CTFFR rejection rate was $31 \%$ in the NXT trial. ${ }^{12}$

Our results are similar to the ADVANCE multicentre registry, which is the largest study to date assessing the clinical efficacy of CTFFR. ${ }^{7}$ It enrolled 5,083 patients who had some degree of atherosclerosis on CTCA and recorded their symptoms, CTCA and CTFFR findings as well as outcome at 90 days. Overall, $72.3 \%$ of patients undergoing ICA with CTFFR $\leq 0.80$ were revascularised. No death or myocardial infarction within 90 days occurred in patients with CTFFR $>0.80(n=1,529)$. Conversely, 19 (0.6\%) major adverse cardiac events (MACE) and $14(0.3 \%)$ cases of death / myocardial infarction occurred in subjects with a CTFFR $\leq 0.80$, which was statistically significant. The authors concluded that CTFFR modified treatment recommendation in two-thirds of subjects compared with CTCA alone and was associated with less negative ICA, predicted revascularisation and also identified subjects at low risk of adverse events. A recent detailed review of CTFFR by Khav et al stated that, while the evidence base for CTFFR is developing, further large-scale studies with cost-benefit analysis and long-term outcomes data are required to justify the additional costs of CTFFR in the Australian health care system. ${ }^{13}$ Our own cost analysis demonstrates that CTFFR can be implemented in a more economical way with a $£ 194.56$ saving PP if CTFFR is only undertaken in patients with $>50 \%$ CTCA stenosis as per ESC guidance. However, assuming that SE accuracy is equal to CTFFR, it can perform the same gatekeeping role at a further cost saving of $£ 228.74$ per patient.

The argument that imaging functional tests such as SE may prove a more cost-effective option is further reinforced by scrutinising the health economic data supporting CTFFR in the PLATFORM trial. ${ }^{14}$ PLATFORM compared CTCA plus CTFFR with the standard of care in patients with stable chest pain. The end point of the study was reduction of ICA showing no significant CAD. The patients were divided into a planned invasive sub-study $(n=380)$ and a planned non-invasive sub-study $(n=204)$. In the CTCA arm of the planned invasive sub-study, CTCA plus CTFFR reduced the rate of ICA as only patients with significant stenosis and positive CTFFR went on to have ICA while, in the ICA arm, all patients were mandated to undergo ICA \pm invasive FFR. In the planned non-invasive sub-study, there was no difference in the rate of ICA between imaging ischaemia tests and CTCA plus CTFFR arms. ${ }^{14}$ However, the health economics evaluation was based on the whole study and not on the sub-studies. ${ }^{15}$ It is, however, important to consider that HeartFlow have recently reduced the UK price of CTFFR to $£ 530$, making it competitive with the more expensive imaging stress tests, such as stress perfusion cardiac magnetic resonance imaging, but not with SE for which the UK national tariff is only $£ 177$. In another detailed review of CTFFR, Nazir et al point out that, despite the projected cost saving across the whole patient pathway as determined by the NICE guideline and the PLATFORM study, local funding for CTFFR will need to come from commissioners, with little available hard evidence that demonstrates any actual downstream savings. ${ }^{16}$
Finally, it is important to consider that the recent publication of the ISCHEMIA trial $(n=5,179)$ may potentially change clinical practice once more. ${ }^{17}$ ISCHEMIA was the largest clinical trial randomising patients with chest pain and moderate to severe ischaemia on stress testing to optimal medical therapy (OMT) versus OMT plus invasive management. The patients were mandated to have a CTCA to exclude significant left main stem (LMS) stenosis before randomisation. ISCHEMIA found no difference in the combined primary outcome of myocardial infarction, resuscitated cardiac arrest, unstable angina, heart failure and death between the two strategies. However, there was a reduction in angina in the invasive strategy and $26 \%$ of patients in the OMT arm crossed over to invasive management during the 4 year follow-up, with $21 \%$ revascularised. Thus, most patients with new stable chest pain need only a diagnostic CTCA, to investigate for the presence of CAD and exclude significant LMS stenosis. The ISCHEMIA trial results mean that the requirement for second-line tests to assess for the significance of coronary stenoses is likely to decrease significantly.

\section{Limitations}

This is a small registry from a single NHS trust comprising two hospitals utilising a relatively novel technology. The initial lack of familiarity with CTFFR may have influenced the number of patients being referred for further evaluation with SE to confirm CTFFR results. This may change as further evidence emerges and familiarity of the technology develops. We note CTCA images needed to be of high quality for CTFFR analysis to be possible, potentially introducing an element of bias. Furthermore, only small numbers of patients underwent invasive FFR, limiting assessment of the comparison with CTFFR on a per vessel basis.

\section{Conclusion}

CTFFR can potentially be an effective rapid assessment of the functional significance of coronary stenoses found on CTCA, but clinical judgment is needed for patients with borderline CTFFR values. CTFFR has practical advantages compared with SE in terms of expediency of test results. However, SE is a lower-cost test. Importantly, the recent ISCHEMIA trial results may move the goalposts again, reducing the need for assessment of functional significance of coronary stenoses found on CTCA.

\section{References}

1 Montalescot G, Sechtem U, Achenbach S et al. 2013 ESC guidelines on the management of stable coronary artery disease: the task force on the management of stable coronary artery disease of the European Society of Cardiology. Eur Heart ] 2013;34:29493003.

2 National Institute for Health and Care Excellence. Addendum to Clinical Guideline (CG95), Chest pain of recent onset: Assessment and diagnosis. NICE, 2016.

3 Meijboom WB, Meijs MFL, Schuijf JD et al. Diagnostic accuracy of 64-slice computed tomography coronary angiography: a prospective, multicenter, multivendor study. J Am Coll Cardiol 2008:52:2135-44.

4 Budoff MJ, Dowe D, Jollis JG et al. Diagnostic performance of 64-multidetector row coronary computed tomographic angiography for evaluation of coronary artery stenosis in individuals without known coronary artery disease: results from the prospective multicenter ACCURACY (Assessment by 
Coronary Computed Tomographic Angiography of Individuals Undergoing Invasive Coronary Angiography) trial. J Am Coll Cardiol 2008;52:1724-32.

5 Siontis GC, Mavridis D, Greenwood JP et al. Outcomes of non-invasive diagnostic modalities for the detection of coronary artery disease: network meta-analysis of diagnostic randomised controlled trials. BMJ 2018;360:k504.

6 National Institute for Health and Care Excellence. HeartFlow FFRCT for estimating fractional flow reserve from coronary CT angiography: Medical technologies guidance [MTG32]. NICE, 2017.

7 Fairbairn TA, Nieman K, Akasaka T et al. Real-world clinical utility and impact on clinical decision-making of coronary computed tomography angiography-derived fractional flow reserve: lessons from the ADVANCE registry. Eur Heart J 2018;39:3701-11.

8 Curzen N, Rana O, Nicholas Z et al. Does routine pressure wire assessment influence management strategy at coronary angiography for diagnosis of chest pain: the RIPCORD study. Circ Cardiovasc Interv 2014;7:248-55.

9 Nam J, Briggs A, Layland J et al. Fractional flow reserve (FFR) versus angiography in guiding management to optimise outcomes in non-ST segment elevation myocardial infarction (FAMOUSNSTEMI) developmental trial: cost-effectiveness using a mixed trial- and model-based methods. Cost Eff Resour Alloc 2015;13:19.

10 Nørgaard BL, Gaur S, Leipsic ] et al. Influence of coronary calcification on the diagnostic performance of $\mathrm{CT}$ angiography derived FFR in coronary artery disease: a substudy of the NXT trial. JACC Cardiovasc Imaging 2015;8:1045-55.

11 Fyyaz S, Hudson J, Olabintan O et al. Computed tomography coronary angiography: Diagnostic yield and downstream testing. Clin Med 2020;20:81-5.
12 Nørgaard BL, Leipsic J, Gaur S et al. Diagnostic performance of noninvasive fractional flow reserve derived from coronary computed tomography angiography in suspected coronary artery disease: the NXT trial (Analysis of Coronary Blood Flow Using CT Angiography: Next Steps). J Am Coll Cardiol 2014;63:1145-55.

13 Khav N, Ihdayhid AR, Ko B. CT-derived fractional flow reserve (CTFFR) in the evaluation of coronary artery disease. Heart Lung Circ 2020;29:1621-32.

14 Douglas PS, Pontone G, Hlatky MA et al. Clinical outcomes of fractional flow reserve by computed tomographic angiography-guided diagnostic strategies vs. usual care in patients with suspected coronary artery disease: the prospective longitudinal trial of FFR(CT): outcome and resource impacts study. Eur Heart J 2015;36:3359-67.

15 Douglas PS, De Bruyne B, Pontone G et al. 1-year outcomes of FFRCT-guided care in patients with suspected coronary disease: the PLATFORM study. J Am Coll Cardiol 2016;68:435-45.

16 Nazir MS, Mittal TK, Weir-McCall J et al. Opportunities and challenges of implementing computed tomography fractional flow reserve into clinical practice. Heart 2020;106:1387-93.

17 Maron DJ, Hochman JS, Reynolds HR et al. Initial invasive or conservative strategy for stable coronary disease. N Engl J Med 2020;382:1395-407.

Address for correspondence: Dr Khaled Alfakih, University Hospital Lewisham, Lewisham High Street, London SE13 6LH, UK.

Email: khaled.alfakih@nhs.net 